\title{
Hypermnesia and the organization of recall
}

\author{
SCOTT C. DAVIS and ROGER L. DOMINOWSKI \\ University of Illinois, Chicago, Illinois
}

\begin{abstract}
In this study, the role of organizational processes in hypermnesia was investigated. Subjects were given 40 simple riddles and asked to recall their answers on each of five consecutive recall trials. Two measures of organization, based on interitem response times during recall or on repetition of item pairings between successive recall trials, were used. The two measures yielded comparable findings. Total recall and organized recall increased across recall trials, but unorganized recall did not. The findings support the hypothesis that the construction of a relatively stable, interitem organizational scheme is important for the growth of recall. It was suggested that organizational processes may contribute to the maintenance of items once recalled rather than to their initial retrieval.
\end{abstract}

Hypermnesia has been characterized as an increase in recall over time (e.g., Shapiro \& Erdelyi, 1974). Previous work focused on demonstrating increases in recall and on the question of whether hypermnesia is more probable with some kinds of materials than with others. Recall increases have been observed for a variety of materials with varying degrees of consistency (e.g., Belmore, 1981; Buschke, 1974; Erdelyi \& Becker, 1974; Erdelyi, Finkelstein, Herrell, Miller, \& Thomas, 1976; Madigan \& Lawrence, 1980; Roediger \& Thorpe, 1978).

Roediger and Payne (1982) made an important contribution by demonstrating that recall increased with repeated testing but not with the passage of time per se. In effect, their findings require that hypermnesia be defined as an increase in recall over repeated attempts at retrieval, and they focus attention on how repeated retrieval might lead to better recall. Roediger and Payne (1982) suggested that hypermnesia might be explained in terms of the construction of alternative retrieval routes or the strengthening of associations between the retrieval context and the recalled items.

The present study was designed to investigate processes contributing to increased recall over repeated attempts, but from a different perspective. For traditional multipletrial, free-recall tasks, where study and recall periods alternate, organizational processes have been shown to play a central role in accounting for the learning that takes place. As (study-recall) trials continue, subjects organize the material, construct larger units linking multiple list items, and thereby increase the number of items recalled (see Tulving, 1968). It is reasonable to propose that hypermnesia may involve comparable organizational processes. With the standard study-test procedure,

A partial report of these data was made at the annual meeting of the Midwestern Psychological Association, Chicago, May, 1981. Requests for reprints should be sent to Roger L. Dominowski, Department of Psychology, University of Illinois at Chicago, Box 4348, Chicago, IL 60680 . repeated recall, rather than repeated study, seems to play the major role in developing organization. Increased organization is illustrated by increasing similarity of successive recalls regarding word order, whereas the order in which items are recalled, especially after several trials, is quite different from presentation orders during study periods (Tulving, 1968). Therefore, a series of recall attempts without intervening study periods may be sufficient to develop organizational structures and increase the amount of recall.

In the present study, subjects were asked to solve a series of simple riddles having one-word answers and were told that they would later be asked to recall the answer words. These materials were used because they have been found to yield a clear hypermnesia effect (Erdelyi, Buschke, \& Finkelstein, 1977). Subjects made five consecutive attempts to recall the words. In addition to assessing the number of words correctly recalled on each attempt, two measures of recall organization were obtained. One was based on the similarity of successive recall protocols, specifically the recurrence of adjacent pairs of words on consecutive protocols, called pair frequency by Sternberg and Tulving (1977). In essence, the repetition of two words in adjacent recall positions, in either order, contributes to the measure of organization. This measure was calculated for Recall Trials 2-5, in each case by comparing the recall protocol to that from the immediately preceding trial. The second measure employed interresponse time intervals and was used by Chase and Simon (1973) and by Reitman (1976) to study memory in the games of chess and Go, respectively. Its logic is that recall items belonging to the same "chunk" will have short interresponse times, whereas recall of items of different chunks will be separated by longer pauses. The previous investigators had found that $2 \mathrm{sec}$ was a satisfactory criterion, and the same criterion was used here. Thus, items recalled within $2 \mathrm{sec}$ of each other were considered to belong to the same chunk and to contribute to the measure of organized recall. The two measures were used to provide some convergence for assessing recall or- 
ganization. Although they have different properties (the temporal measure can be applied to all trials and can reflect changes in trial-to-trial organization, whereas pair repetition cannot), it was expected that they would have similar relations to total recall. If increased organization is involved in hypermnesia, we would expect to find increases in total recall and in both organizational measures across recall trials.

\section{METHOD}

\section{Subjects}

The subjects were 21 students who elected to participate in this study as partial fulfillment of a requirement of an introductory psychology course.

\begin{abstract}
Materials
Forty simple riddles constructed to have single-word answers were employed. All answers were object names. A pilot study demonstrated that the riddles were easy to solve; two examples follow: "This longish object is used by baseball players to hit the ball." "This is worn on the wrist and is used to tell time." Answers are "bat" and "watch," respectively.

\section{Design and Procedure}

The basic design was to have subjects work through the riddles once, then attempt to recall the answers on five consecutive recall trials. Subjects were instructed to read each riddle as it was presented and to try to remember the object the riddle described, because they would be asked to recall the object's name later. An example was given; the riddles were then presented via memory drum at the rate of $5 \mathrm{sec}$ per riddle. After the riddles had been presented, subjects were asked to recall the object names, speaking into a microphone. Five min were allowed for each recall trial, and the five trials followed one another consecutively. All recall trials were tape-recorded; interresponse times were found with the use of an oscillograph record of the impulses on tape.
\end{abstract}

\section{RESULTS}

Changes across recall trials were analyzed for total correct recall and for both measures of organization. For temporal organization, for each trial, organized groups were defined as items having interresponse times of less than $2 \mathrm{sec}$; the total number of words recalled in all groupsexcluding the first item in each group-was the organizedrecall score for that trial. For pair repetition, for trials $2-5$, groups were defined as consecutively recalled items that had occurred in adjacent positions, in either order, on the previous trial. Again excluding the first item in each group, the total number of words recalled in all groups was the organized-recall score for the trial in question. The rationale for not counting the first word in a group as remembered via organized recall is that this item is presumably recalled by some means other than linkage with other recalled items (e.g., Tulving, 1962). The pairrepetition measure reported here is equal to Sternberg and Tulving's (1977) pair-frequency measure without the correction for "estimated chance pairings." It should be noted that employing the correction does not change the results reported here; the means for Sternberg and Tulving's measure, for Trials 2-5, were 2.2, 3.8, 4.7, and 5.7, respectively. The two measures reported here con- veniently allow total recall to be divided into the numbers of organized and unorganized words.

Extralist intrusions occurred infrequently, with no change across recall trials; there was an average of 0.98 intrusion per subject per trial. Because subjects had no record of what they had already recalled on a trial, they sometimes repeated correct items. Only the first recall of an item was used in calculating total recall or the measures of organization. Linear changes across trials were examined; all effects reported were significant, with $\alpha=.01$.

Mean values for total recall and the various measures derived from recall protocols are shown in Table 1. Hypermnesia was observed; total recall increased linearly across trials $[F(1,20)=92.93, M S e=1.39]$. Both measures of organized recall also exhibited linear increases over trials [for temporal organization, $F(1,20)=106.70$, $M S \mathrm{e}=2.02$; for pair repetition, $F(1,20)=24.40$, $M S e=5.81]$. In contrast, unorganized recall did not increase over trials. Relative to pair repetition, there was no change in unorganized recall across trials, whereas for the temporal measure, there was a tendency for unorganized recall to decline $[F(1,20)=5.20, M S e=2.07$, $p<.05]$.

Table 1 also shows, for both organizational measures, the average numbers of organized groups found on each trial. For both measures, the number of groups increased over trials [for temporal organization, $F(1,20)=22.07$, $M S \mathrm{e}=1.63$; for pair repetition, $F(1,20)=17.53$, $M S e=1.96]$. An index of group size may be obtained by dividing the number of organized words by the number of groups; average group size increased slightly over trials. Relative to temporal organization, average group size was definitely smallest on Trial 1 , but there were no further increases on later trials (and a slight decline on Trial 5). Relative to the pair-repetition measure, average group size showed a slight but consistent increase over Trials 2-5.

Although both organizational measures had very similar relations to recall trials, they are, of course, not iden-

Table 1

Means For Recall Measures Across Trials

\begin{tabular}{lrrrrr}
\hline & \multicolumn{5}{c}{ Trial Number } \\
\cline { 2 - 6 } Measure & \multicolumn{1}{c}{1} & \multicolumn{1}{c}{3} & \multicolumn{1}{c}{4} & 5 \\
\hline Total Number of Words & 18.1 & 18.9 & 20.4 & 21.6 & 22.5 \\
Temporal Organization & & & & & \\
$\quad$ Organized Words & 7.0 & 9.7 & 11.8 & 12.5 & 12.9 \\
$\quad$ Unorganized Words & 11.1 & 9.2 & 8.6 & 9.1 & 9.6 \\
$\quad$ Number of Groups & 2.7 & 3.0 & 3.6 & 3.9 & 4.3 \\
$\quad$ Pair Repetition & & & & & \\
$\quad$ Organized Words & & 3.6 & 5.4 & 6.2 & 7.3 \\
$\quad$ Unorganized Words & & 15.4 & 15.0 & 15.4 & 15.2 \\
$\quad$ Number of Groups & & 3.1 & 4.3 & 4.5 & 5.0 \\
Changes Between Trials & & & & & \\
$\quad$ Words Retained & & 16.0 & 17.8 & 18.9 & 20.7 \\
$\quad$ Words Lost & 2.1 & 1.2 & 1.5 & 0.9 \\
$\quad$ "New" Words & 2.9 & 2.6 & 2.7 & 1.8 \\
\hline
\end{tabular}


tical. The temporal measure identifies more words as organized and tends to show fewer, larger groups. The two measures do, however, agree to a reasonable degree; the data for Trial 5 were examined with the result that $86 \%$ of the individual items identified as organized by pair repetition were also organized using the temporal measure.

With respect to total recall, the primary finding was an increase across recall trials. There were, however, fluctuations from trial to trial in the status of individual items. The bottom part of Table 1 shows, for Trials 2-5, the numbers of items retained or lost from the previous trial as well as the number of "new" items recalled (words not recalled on the immediately preceding trial). Across trials, there was a linear increase in the number of words retained from the previous trial $[F(1,20)=72.93$, $M S e=3.20]$. The number of new words recalled showed a decline across trials $[F(1,20)=13.54, M S e=0.92]$, whereas there was a nonsignificant decline in words lost from the previous trial. Recall had not reached asymptote after five trials; the mean total number of words recalled on Trial 5 (22.5) was far short of the maximum possible (40) and less than the number of different words recalled at least once during the recall trials (25.0).

The increases observed across trials were quite consistent among subjects. The percentages of individual subjects showing positive linear slopes for the several measures were: total recall, $100 \%$; temporal organization, $100 \%$; pair repetition, $85 \%$; words retained from the previous trial, $100 \%$. Despite the relatively restricted ranges of the measures, supplementary correlational analyses produced several findings worthy of mention. Variation in linear increases in total recall was related neither to total recall nor to temporally organized recall on Trial 1 , but tended to be inversely related to the number of unorganized words recalled on Trial 1 [ $r=-0.49]$. For the five trials, total recall was more strongly related to temporally organized recall (mean $r=+0.72$ ) than to unorganized recall (mean $r=+0.52$ ). For Trials 2-5, the number of words retained from the previous trial was more strongly related to organized recall on the prior trial (mean $r=+0.70$ ) than to unorganized recall on the prior trial (mean $r=+0.46$ ).

\section{DISCUSSION}

The results support the hypothesis that organizational processes may play an important role in hypermnesia. Total recall and organized recall increased over recall trials, but unorganized recall did not. This statement is true for both the measures of organization, which strengthens the finding. The correlational results support a connection between organization and recall growth. Organized recall is superior to unorganized recall as a predictor of total recall on a trial and of the number of words that will be retained on the following trial.

Organizational processes do not account for all of recall-many items are recalled with no indication of interitem organization. Nonetheless, increased organization is associated with increased recall. Rather than supporting explanations of hypermnesia in terms of "strengthened" contextual associations or the development of alternative item encodings, the findings suggest that the construction of a relatively stable interitem retrieval scheme is important (see Young \& Bellezza, 1982). Put simply, any proposed mechanism that applies to single items would have difficulty explaining why organized recall, but not unorganized recall, increases over trials.

It is obvious that, for hypermnesia to occur, new items must be recalled over successive recall attempts. It is important to realize, however, that successive recalls are unstable, with some items retained and others lost from the previous trial. Recalling new items will not by itself produce hypermnesia. One possible way for total recall to increase is as follows: Constant numbers of items are retained and lost between trials, but the number of new items increases. The present data contradict this notion; recall of new items did not increase, but decreased over trials, and retention of items between trials increased. This suggests that hypermnesia occurs through the retention of an increasing number of words from trial to trial while some new items are recalled.

Similarly, interitem organization need not lead to increased recall. That is, if a fixed set of items became organized, it might be well maintained over successive recalls, but this might lead only to stability rather than to growth. For total recall to increase, new items must be retrieved and then maintained over successive recalls. One possibility is that the construction of an organized group (of previously recalled items) provides a better alternative retrieval path for previously unrecalled items. The present data do not support the proposal of this mechanism, however. On Trial 2, only $13 \%$ of new items were organized, according to the temporal measure (the definition of pair repetition makes all new items unorganized). These items were well-maintained over Recall Trials 3-5 (90\% recall) and tended to occur in organized groups on these trials ( $73 \%$ by temporal organization, $58 \%$ by pair repetition). It thus appears that interitem organization, rather than facilitating the initial retrieval of new items, contributes to their maintenance over successive recalls and thus to increased total recall.

This account implies that, for hypermnesia to occur, items must be sufficiently well-encoded that they can be retrieved at least once on the basis of cues other than interitem relations. Once recalled, organization processes provide the retrieval cues for their being maintained over successive recall attempts. From this perspective, deficiencies in either item memorability or organizational opportunities should lead to the reduction or elimination of hypermnesia.

\section{REFERENCES}

BelmoRe, S. M. (1981). Imagery and semantic elaboration in hypermnesia for words. Journal of Experimental Psychology: Human Learning \& Memory, 7, 191-203.

BUSCHKE, H. (1974). Spontaneous remembering after recall failure. Science, 184, 579-581.

Chase, W. G., Simon, H. A. (1973). Perception in chess. Cognitive Psychology, 4, 55-81.

ERDELYI, M. H., \& BECKER, J. (1974). Hypermnesia for pictures: Incremental memory for pictures but not words in multiple recall trials. Cognitive Psychology, 6, 159-171.

ERdelyi, M. H., BuschKe, H., \& Finkelstein, S. (1977). Hypermnesia for Socratic stimuli: The growth of recall for an internally generated memory list abstracted from a series of riddles. Memory \& Cognition, 5, 283-286.

Erdelyi, M. H., Finkelstein, S., Herrell, N., Miller, B., \& Thомаs, J. (1976). Coding modality vs. input modality in hypermnesia: Is a rose a rose a rose? Cognition, 4, 311-319.

Madigan, S., \& LAWRENCE, V. (1980). Factors affecting item recovery and hypermnesia in free recall. American Journal of Psychology, 93, 489-504.

ReItman, J. S. (1976). Skilled perception in Go: Deducing memory structure from inter-response times. Cognitive Psychology, 8, 336-356.

Roediger, H. L., \& PAYNe, D. G. (1982). Hypermnesia: The role of repeated testing. Journal of Experimental Psychology: Learning, Memory, \& Cognition, 8, 66-72.

RoEDiger, H. L., \& ThORPE, L. A. (1978). The role of recall time in producing hypermnesia. Memory \& Cognition, 6, 296-305. 
Shapiro, S. R., \& ERdelyi, M. H. (1974). Hypermnesia for pictures but not words. Journal of Experimental Psychology, 103, 1218-1219.

Sternberg, R. J., \& Tulving, E. (1977). The measurement of subjective organization. Psychological Bulletin, 84, 539-556.

TulviNG, E. (1962). Subjective organization in free recall of "unrelated" words. Psychological Review, 69, 344-354.
Tulving, E. (1968). Theoretical issues in free recall. In T. R. Dixon \& D. L. Horton (Eds.), Verbal behavior and general behavior theory. Englewood Cliffs, NJ: Prentice-Hall.

Young, D. R., \& BellezzA, F. S. (1982). Encoding variability, memory organization, and the repetition effect. Journal of Experimental Psychology: Learning, Memory, \& Cognition, 8, 545-559.

(Manuscript received for publication July 15, 1985.) 